\title{
As ações de sentar e levantar do solo são prejudicadas por excesso de peso
}

\author{
Vitor Agnew Lira ${ }^{1}$, Elirez Bezerra da Silva ${ }^{1,2}$ e Claudio Gil Soares de Araújo ${ }^{1,3}$
}

\section{RESUMO}

Inatividade física é comum em pessoas com sobrepeso. O objetivo do presente trabalho foi identificar o efeito agudo do incremento no peso corporal sobre o desempenho nas ações de sentar e levantar do solo e verificar como o mesmo sofria influência de algumas variáveis morfofuncionais. Preliminarmente (E1), 33 soldados, com idade de $20 \pm 1,4$ anos (média $\pm \mathrm{dp}$ ) e valores homogêneos de IMC $\left(22 \pm 1,0 \mathrm{~kg} / \mathrm{m}^{2}\right)$ e $\% \mathrm{~g}(5,3 \pm 2,3)$, foram divididos aleatoriamente em três grupos com 11 integrantes. O Teste de Sentar-Levantar (TSL) foi aplicado, em uma ordem quadrada latina, sem incremento artificial no peso (A0), e com $10 \%$ (A10) e $20 \%$ de incremento (A20), simulados através da utilização de coletes de areia localizados no tronco. No segundo estudo (E2), 24 jogadores de futebol púberes e pós-púberes (15,4 $\pm 1,1$ anos), aleatoriamente divididos em quatro grupos de seis e com flexibilidade geral similar, medida através do Flexiteste, foram submetidos ao TSL, como em E1, mas também na condição de $30 \%$ de incremento no peso (A30). Entre os indivíduos cujo desempenho, em cada ação, se mantinha máximo com o aumento no peso e os demais, compararam-se as flexibilidades geral e específica por movimento em E1, e IMC, $\Sigma 7$ dobras cutâneas, \%g, relações cintura-quadril, cintura-coxa e entre dobras cutâneas superiores e inferiores, somatotipo de Heath-Carter, potência de membros inferiores (salto vertical) e tempo em corrida de 50m, em E2. No sentar, o teste de Friedman não identificou diferenças significativas dentre as condições em E1 $(p=0,21)$ e em E2 $(p=0,07)$.

\footnotetext{
1. Programa de Pós-Graduação em Educação Física da Universidade Gama Filho.

2. Escola de Educação Física do Exército.

3. Clinimex - Clínica de Medicina do Exercício, Rio de Janeiro, RJ, Brasil. Recebido em: 10/6/2000

Aceito em: 1/11/2000
}

Endereço para correspondência:

Rua Siqueira Campos, 93/101

22031-070 - Rio de Janeiro, RJ - Brasil

E-mail: cgaraujo@iis.com.br

Rev Bras Med Esporte - Vol. 6, № 6 - Nov/ Dez, 2000
Mas essas ocorreram, para o levantar, em ambos os estudos ( $\mathrm{p}<0,001$ e $\mathrm{p}=0,03$ ). O teste de Tukey demonstrou que somente incrementos de $20 \%$ no peso eram suficientes para deteriorar o desempenho em E1 ( $p<0,05)$, e de 30\% em E2 $(p<0,05)$. Em E1, verificou-se que os indivíduos que mantinham desempenho máximo para o sentar eram mais flexíveis na adução de quadril ( $\mathrm{p}<0,001)$ e, para o levantar, na flexão do quadril $(\mathrm{p}=0,02)$. Em E2, não houve diferença significativa nas variáveis. Provavelmente, os indivíduos com sobrepeso e obesos enfrentam maiores dificuldades nas atividades realizadas em oposição à força da gravidade, como o levantar. Esses achados e a influência da flexibilidade devem ser confirmados em estudos futuros com essa população.

Palavras-chave: Teste de Sentar-Levantar. Sobrepeso. Obesidade. Flexibilidade. Variáveis morfofuncionais. Avaliação funcional.

\section{ABSTRACT}

Actions of sitting and rising from the ground are negatively affected by overweight

Physical inactivity is common in overweight individuals. The objective of this study was to identify the acute effect of body weight increase on the performance of sitting and rising actions from the ground, and to verify the influence of some morphologic and functional variables on these movements. Initially (E1), 33 soldiers, aged $20 \pm 1.4$ years (mean $\pm s d$ ) and with similar values of BMI $(22 \pm 1.0 \mathrm{~kg} / \mathrm{m} 2)$ and $B F \%(5.3 \pm 2.3)$, were randomly divided into three groups of 11 subjects. The Sitting-Rising Test (SRT) was applied in a latin square order without artificial increment in the weight (AO), and with $10 \%$ (A10), and 20\% of increment (A20), simulated by the use of sand vests placed in the trunk. In the second study (E2), 24 pubescent and post-pubescent soccer players (15.4 \pm 1.1 years), randomly divided into four groups of six and with similar general flexibility, measured by the Flexitest, were submitted to SRT as in E1, but also in the condition of $30 \%$ increment in body weight (A30). Between individuals who maintained the maximal score in each action with the 
increase in weight and the others, general and specific movement flexibilities were compared in E1, and BMI, $\Sigma 7$ skinfolds, BF\%, waist-hip and waist-thigh and superior to inferior skinfolds ratios, Heath-Carter somatotype, leg muscle power (vertical jump), and $50 \mathrm{~m}$ sprint time, in E2. In sitting, Friedman test did not identify differences among the conditions in both E1 $(p=.21)$ and E2 $(p=.07)$. But for rising they occurred in both studies $(p<.001$ and $p=$ .03). Tukey test showed that only a $20 \%$ weight increment was sufficient to deteriorate the performance in El ( $p<$ $.05)$, and $30 \%$ in E2 ( $p<.05)$. In E1, it was verified that individuals who maintained maximal score for sitting were more flexible in hip adduction $(p<.001)$, and those for rising in hip flexion $(p=.02)$. In E2, no significant differences were found in the variables. Likely, obese and overweight individuals face more difficulties in the activities accomplished against gravity force, as rising. Those findings and the influence of flexibility should be corroborated in future studies using this population.

Key words: Sitting-Rising Test. Overweight. Obesity. Flexibility. Morphological and functional variables. Functional evaluation.

\section{INTRODUÇÃO}

Sobrepeso ou excesso de peso corporal, definido como índice de massa corporal (IMC) superior a $25 \mathrm{~kg} / \mathrm{m}^{2}$ ou por percentuais de gordura $(\% \mathrm{~g})$ acima de 20 , em homens jovens ${ }^{1}$, vem apresentando prevalência crescente na sociedade moderna ${ }^{2}$. Atualmente, a população americana está mais pesada do que nunca ${ }^{3,4}$, e esta parece ser uma tendência não só de países desenvolvidos, mas também de países em desenvolvimento ${ }^{5,6}$. Sabidamente, o sobrepeso é um fator de risco para doenças crônico-degenerativas como a hipertensão arterial, aterosclerose e o diabetes mellitus ${ }^{7,8}$. Baixos níveis de atividade física são uma das características prevalentes em pessoas com sobrepeso e obesidade, aspecto que acaba por adicionar riscos à saúde ${ }^{9,10}$. Isto posto, parece lógico que os indivíduos com sobrepeso apresentem maiores dificuldades em realizar tarefas simples do dia-a-dia, embora esse aspecto pareça não ter sido ainda contemplado formalmente em estudos científicos.

Tarefas simples como sentar e levantar de uma cadeira têm sido utilizadas para estimar o risco de queda em ido$\operatorname{sos}^{11-13}$. Com o propósito de acrescentar mais dificuldade às ações, aprimorando a sensibilidade das mesmas na avaliação de outras populações, Araújo ${ }^{14,15}$ desenvolveu o Teste de Sentar-Levantar (TSL). O teste avalia o desempenho nas ações de sentar e levantar do solo, sendo possivelmente útil para avaliar a aptidão física funcional de indivíduos em várias faixas etárias. Em estudo prévio, verificou-se que o teste apresenta elevadas fidedignidades interavaliadores, interdias e intradia, com concordância absoluta entre avaliadores, ou manutenção dos escores individuais em avaliações seguidas, de 80 a $76 \%$ para as ações de sentar e levantar, respectivamente ${ }^{16}$. Ainda se observou que os escores atribuídos por distintos avaliadores, ou obtidos em avaliações individuais próximas, quando variam, o fazem minimamente dentro da escala de medida para ambas as ações (15 e 16\%, respectivamente). O objetivo da presente investigação foi identificar o efeito agudo do incremento no peso corporal sobre o desempenho em sentar e levantar do solo.

\section{MÉTODOS}

Desenvolveram-se dois estudos com amostras distintas. Primeiramente, foi conduzido um estudo preliminar envolvendo soldados com padrões semelhantes de atividade física, apresentando pequena variação no percentual de gordura $(\% \mathrm{~g})$ e no índice de massa corporal (IMC). Avaliou-se a flexibilidade nas articulações de tornozelo, joelho, quadril e tronco, bem como o desempenho no TSL em condições sem interferência (controle) e à medida que o peso corporal era incrementado (estudo 1). Posteriormente, avaliaram-se jogadores de futebol adolescentes, com maiores níveis de atividade física aeróbia, mas com padrões homogêneos de flexibilidade nas mesmas articulações medidas no estudo 1. Embora o desempenho nas ações de sentar e levantar tenha sido avaliado também a exemplo do estudo 1 , outras variáveis foram quantificadas nesta abordagem, a saber: IMC, \%g, somatório de dobras cutâneas, somatotipo, relações entre as circunferências de cintura e quadril (R.C/Q) e de cintura e coxa superior (R.C/Cx), relação entre dobras cutâneas superiores e inferiores (RDCSI) e a potência muscular de membros inferiores (estudo 2).

\section{Amostras}

Para controlar a influência de idade, sexo, IMC e nível de atividade física, conduziram-se duas abordagens isoladas, porém semelhantes, envolvendo amostras distintas. A participação dos indivíduos era voluntária. Nenhum dos indivíduos avaliados apresentava sintomatologia para o sistema locomotor. No estudo preliminar, foram observados 33 soldados, com IMC entre 20 e $23 \mathrm{~kg} / \mathrm{m}^{2}$, que realizavam exercícios predominantemente aeróbios durante uma hora, em três sessões semanais. Os indivíduos apresentavam idade, peso, estatura, IMC e Flexíndice reduzido de $20 \pm 1,4$ anos (média $\pm \mathrm{dp}$ ), 65,2 $\pm 6,2 \mathrm{~kg}, 172 \pm 8,0 \mathrm{~cm}, 22 \pm 1,0 \mathrm{~kg} / \mathrm{m}^{2} \mathrm{e}$ $29 \pm 4,2$ pontos, respectivamente. Posteriormente, foram avaliados 24 jogadores de futebol adolescentes, do sexo masculino, que se encontravam em fase de competição. Os mesmos estavam passando por uma rotina de treinamento 
composta de seis sessões semanais de exercícios aeróbios, com duração de duas horas. A amostra era homogênea quanto à idade e flexibilidade geral e específica. Através da escala de Tanner ${ }^{18}$ (1962) para pêlos pubianos, todos os indivíduos se auto-avaliaram como $\mathrm{P} 4$, ou púberes $(\mathrm{n}=11)$, e P5, ou pós-púberes $(\mathrm{n}=13)$. Os valores para idade, peso corporal, estatura, IMC e Flexíndice reduzido foram (média \pm dp): $15,4 \pm 1,1$ anos, $65,3 \pm 7,3 \mathrm{~kg}, 176,3 \pm 5,7 \mathrm{~cm}$, $21 \pm 1,7 \mathrm{~kg} / \mathrm{m}^{2}$ e $25,6 \pm 2,4$ pontos, respectivamente.

\section{Dados antropométricos}

$\mathrm{O}$ peso corporal e a estatura foram medidos em uma balança calibrada e em um estadiômetro, com leituras de $0,5 \mathrm{~kg}$ e $1 \mathrm{~mm}$, respectivamente, sendo os resultados utilizados para o cálculo do IMC. Um compasso (Cescorf, Porto Alegre, Brasil) com leitura de $0,1 \mathrm{~mm}$ foi usado para medir as dobras cutâneas. Considerando um provável padrão andróide de distribuição de gordura corporal, baseando-se nas características de sexo e idade das amostras, as dobras cutâneas peitoral, de abdômen e coxa foram selecionadas para estimar a densidade corporal ${ }^{18}$ e o $\% \mathrm{~g}^{19}$. Nos jogadores de futebol, as dobras cutâneas subescapular, supra-ilíaca, de tríceps e de perna medial também foram quantificadas, utilizando-se o somatório de sete dobras cutâneas $\left(\sum\right.$ dc) como outra variável de adiposidade. O somatotipo de Heath-Carter também foi calculado nos atletas, utilizando equações previamente descritas na literatura ${ }^{20,21}$.

Com o propósito de avaliar a influência da distribuição de gordura corporal sobre o desempenho de sentar e levantar do solo com o peso corporal alterado, observou-se a relação entre as médias das dobras cutâneas superiores e inferiores (RDCSI), como também as relações entre as circunferências de cintura e quadril (R.C/Q) e de cintura e coxa superior (R.C/Cx). Todas as circunferências foram medidas no plano horizontal, usando-se uma fita antropométrica com leitura de $0,1 \mathrm{~mm}^{22}$. A circunferência de cintura foi medida ao nível do umbigo e a maior circunferência glútea considerada como a medida de quadril. A circunferência de coxa superior foi tomada logo abaixo da prega glútea.

\section{Avaliação de flexibilidade}

A flexibilidade estática, definida como a máxima amplitude fisiológica passiva em um dado movimento ${ }^{23}$, foi avaliada de forma passiva através do Flexiteste ${ }^{24}$. Este instrumento envolve a medida da amplitude em 20 movimentos nas sete principais articulações ${ }^{23,24}$. A amplitude de movimento observada é quantificada através da comparação com um mapa de avaliação contendo uma escala progressiva e descontínua de 0 a 4 , com intervalos de um ponto. Considerando que os resultados de todos os movimentos individuais têm uma distribuição gaussiana, é possível adicioná-

Rev Bras Med Esporte - Vol. 6, № 6 - Nov/ Dez, 2000 los obtendo-se um índice adimensional de flexibilidade geral, chamado Flexíndice, cujos resultados podem ser tratados parametricamente. Nos estudos aqui abordados, foram avaliados somente os 11 movimentos das quatro articulações principalmente envolvidas nas ações do TSL. As articulações e respectivos movimentos avaliados foram: tornozelo (flexão e extensão), joelho (flexão e extensão), quadril (flexão, extensão, abdução e adução), e tronco (flexão, extensão e flexão lateral). Um Flexíndice reduzido, variando de 0 a 44 pontos, considerando apenas os 11 movimentos avaliados, foi então determinado. De acordo com achados anteriores, a flexibilidade geral e a apresentada em apenas oito movimentos específicos parecem influenciar o desempenho nas ações de se sentar e levantar do solo $^{25}$. Assim, os resultados de flexibilidade foram analisados de dois modos: flexibilidade geral de tronco e membros inferiores $\left(\sum\right.$ dos resultados nos 11 movimentos - Flexíndice adaptado) e a flexibilidade específica (cada um dos oito movimentos, isoladamente).

\section{Potência muscular de membros inferiores}

Avaliou-se a potência muscular de membros inferiores através de dois métodos indiretos, ou seja, considerando a altura alcançada no salto vertical e o peso corporal do avaliado (equação 1), e o tempo em corrida de $50 \mathrm{~m}^{26}$. O salto vertical foi realizado com o avaliado partindo de uma posição preparatória agachado, com os joelhos completamente fletidos, e então saltando e tocando o ponto mais alto possível em uma escala de medida fixada à parede. O resultado foi estabelecido subtraindo-se a altura máxima do atleta, com o braço estendido e pés no chão, da alcançada durante o teste. Três tentativas foram permitidas, após os atletas serem informados sobre o procedimento, e o melhor resultado considerado para o cálculo posterior da potência muscular.

\section{Equação 1}

Potência pico $(\mathrm{w})=60,7 \times$ (altura de salto $[\mathrm{cm}])+45,3 \times$ (massa corporal $[\mathrm{kg}])-2055$

Sayers et al. ${ }^{27}$

O teste de corrida de $50 \mathrm{~m}$ em velocidade máxima foi aplicado individualmente em cada atleta. Utilizou-se um comando visual (abaixar rapidamente o braço que se encontrava elevado) para iniciar o teste. O registro de tempo era realizado em um cronômetro manual, com leitura de 0,01s. O menor tempo no teste, também em três tentativas, foi considerado para análises posteriores.

\section{Teste de Sentar-Levantar (TSL)}

Todos os indivíduos foram submetidos ao TSL ${ }^{14,15}$. Em descrição breve, o TSL é um procedimento simples que avalia o desempenho nas ações de sentar e levantar do solo. A 
avaliação é realizada separadamente para cada ação, utilizando uma escala ordinal progressiva de 0 a 5 , com intervalos de 0,5 ponto. $O$ escore máximo 5 corresponde à ação de sentar equilibradamente, ou à de levantar, sem a utilização de apoio extra (mão e joelho). Cada apoio extra no chão, ou no próprio corpo, realizado com o propósito de auxiliar o movimento, resulta na subtração de um ponto do escore máximo. Qualquer desequilíbrio perceptível durante as ações resulta na perda de mais 0,5 ponto. Normalmente, são necessárias apenas duas tentativas para identificar-se o melhor desempenho possível do avaliado em cada ação ${ }^{14,25}$.

\section{Protocolo do estudo 1}

Todas as medidas foram realizadas em dois dias distintos. No primeiro dia, foram obtidos os dados antropométricos e de flexibilidade. No segundo dia, o TSL foi executado em três circunstâncias, com intervalo mínimo de um minuto: com o peso corporal real (A0) e com $10 \%$ (A10) e $20 \%$ (A20) de incremento no peso. As avaliações seguiram uma ordem quadrada latina, ou seja, o mesmo número de indivíduos $(\mathrm{n}=11)$, aleatoriamente divididos em três grupos, passando por três sequiências distintas de avaliações (A0-A10-A20; A20-A0-A10; A10-A20-A0) ${ }^{28}$. Coletes de areia, localizados no tronco, foram utilizados para os incrementos no peso. Para identificar em que circunstâncias o sobrepeso era caracterizado, calculou-se o IMC e o \%g para cada avaliação, considerando o peso artificial como gordura. Um padrão conservador para o IMC $\left(>25 \mathrm{~kg} / \mathrm{m}^{2}\right)$ e $\% \mathrm{~g}$ maiores que 20 foram considerados como limites inferiores para o sobrepeso ${ }^{1,29}$.

\section{Protocolo do estudo 2}

Todas as medidas foram coletadas ao longo de três dias. O TSL foi administrado nas mesmas circunstâncias que no estudo 1, exceto por também utilizar um incremento de $30 \%$ no peso corporal (A30), totalizando assim quatro avaliações. A amostra foi dividida aleatoriamente em quatro grupos de seis indivíduos e as avaliações novamente realizadas respeitando um quadrado latino. Também foram utilizados coletes de areia, localizados no tronco, para aumentar artificialmente o peso corporal. Os mesmos critérios de \%g usados no primeiro estudo, em adição a valores específicos de IMC para adolescentes brasileiros (> 23kg/ $\mathrm{m}^{2}$ ), foram utilizados para evidenciar a presença de sobrepeso corporal $^{30}$.

\section{Análise de dados}

Em ambos os estudos, utilizou-se o teste não-paramétrico de Friedman para comparar as medianas dos escores do TSL obtidos em cada avaliação. Ao identificar-se diferen- ças significativas, o teste de Tukey foi utilizado para se conhecer entre quais circunstâncias de avaliação as diferenças ocorreram.

Objetivando-se testar se algumas das variáveis antropométricas, de flexibilidade e potência muscular de membros inferiores exerciam influência nos resultados do sentar e do levantar com aumento do peso, dividiu-se cada amostra entre os que eram capazes de manter o escore máximo na ação de sentar, com o peso artificialmente aumentado, e os que não eram. $\mathrm{O}$ mesmo ocorrendo para o levantar. Em seqüência, compararam-se os resultados de cada variável entre os dois grupos para cada ação. Uma vez que a amplitude de movimento havia sido medida nos dois estudos e que os jogadores de futebol apresentavam valores mais semelhantes para as flexibilidades geral e específica que os soldados, apenas a análise da influência destas variáveis foi realizada a partir da comparação de resultados, dos dois grupos, no estudo 1 . Todas as variáveis foram avaliadas através do teste $t$ simples, e a flexibilidade específica pela estatística do qui-quadrado (tabela de contingência), devido à característica ordinal dos pontos.

Um nível de probabilidade inferior a 5\% para o erro do tipo 1 foi considerado em todas as análises $(\mathrm{p}<0,05)$. Os testes apresentaram elevadas potências em relação à análise do efeito do incremento no peso sobre o desempenho das ações de sentar e levantar ( $p>0,95$ ), levando-se em consideração o número de indivíduos observados e considerando a diferença mínima detectável igual a um ponto na escala de medida do TSL, o que envolve o uso de um apoio. Considerando dois desvios padrões como o mínimo a ser discriminado, os mesmos valores foram observados para o confronto das variáveis entre os grupos caracterizados pelo desempenho em cada ação, com exceção para a análise das flexibilidades geral e específica nos grupos determinados quanto ao desempenho para o sentar, na qual a potência foi um pouco menor $(p>0,75)$.

\section{RESULTADOS E DISCUSSÃO}

Em ambos os estudos, verificou-se um desempenho inicial próximo ao máximo no TSL. A condição de sobrepeso foi alcançada também nos dois estudos, mas com magnitudes distintas de aumentos artificiais no peso corporal. As medianas para cada ação demonstram que a maioria dos indivíduos precisou utilizar no máximo mais um apoio, com o incremento de 20 a $30 \%$ no peso corporal (tabela 1). Entretanto, o peso aumentado resultou em maiores dificuldades na ação de levantar do que na de sentar. Em ambos os estudos, os escores referentes ao sentar não foram significativamente comprometidos ( $\mathrm{p}=0,205$ e $\mathrm{p}=0,072$ [estudo 1 e 2, respectivamente]), enquanto diferenças ocorreram para o levantar $(\mathrm{p}<0,001 ; \mathrm{p}=0,03)$. Este não é um 
TABELA 1

Valores de IMC e percentual de gordura (média $\pm \mathrm{dp}$ ) e os escores

\begin{tabular}{|c|c|c|c|c|}
\hline & $\begin{array}{l}\text { Avaliação 1* } \\
\text { (A0) }\end{array}$ & $\begin{array}{c}\text { Avaliação } 2^{\dagger} \\
\text { (A10) }\end{array}$ & $\begin{array}{l}\text { Avaliação } 3^{\ddagger} \\
\text { (A20) }\end{array}$ & $\begin{array}{c}\text { Avaliação } 4^{\S} \\
\text { (A30) }\end{array}$ \\
\hline \multicolumn{5}{|l|}{ Estudo 1} \\
\hline $\mathrm{IMC}\left(\mathrm{kg} / \mathrm{m}^{2}\right)$ & $22 \pm 1$ & $24,2 \pm 1,1$ & $26,4 \pm 1,2$ & - \\
\hline$\% g$ & $5,3 \pm 2,3$ & $13,9 \pm 2,1$ & $21 \pm 1,9$ & - \\
\hline Sentar & 4,5 & 4,5 & 4,5 & - \\
\hline Levantar" & 5,0 & 4,5 & $4,0^{4}$ & - \\
\hline \multicolumn{5}{|l|}{ Estudo 2} \\
\hline $\mathrm{IMC}\left(\mathrm{kg} / \mathrm{m}^{2}\right)$ & $21 \pm 1,7$ & $23 \pm 1,8$ & $25,2 \pm 2$ & $27,3 \pm 2,1$ \\
\hline$\% g$ & $6,5 \pm 1,7$ & $15 \pm 1,5$ & $22,1 \pm 1,4$ & $28,1 \pm 1,3$ \\
\hline Sentar & 5,0 & 4,5 & 4,5 & 4,5 \\
\hline Levantar" & 4,5 & 4,5 & 4,5 & 4,5 \\
\hline \multicolumn{5}{|c|}{ * Peso corporal real } \\
\hline \multicolumn{5}{|c|}{ † $10 \%$ de incremento no peso corporal } \\
\hline \multicolumn{5}{|c|}{$\ddagger 20 \%$ de incremento no peso corporal } \\
\hline \multirow{2}{*}{\multicolumn{5}{|c|}{$\begin{array}{l}\S 30 \% \text { de incremento no peso corporal } \\
\text { II } p<0,05 \text { (teste de Friedman) }\end{array}$}} \\
\hline & & \multicolumn{3}{|c|}{$\begin{array}{l}\text { II } p<0,05 \text { (teste de Friedman) } \\
\text { diferente da condicão real de peso corporal }(p<0,05-\text { teste de Tukey) }\end{array}$} \\
\hline
\end{tabular}

achado inesperado, já que o movimento de levantar de uma cadeira correlaciona-se com a força de membros inferiores ${ }^{13,25}$, e à medida que o peso é aumentado há maior solicitação motora para vencer a resistência do mesmo. A força da gravidade, atuando em oposição aos movimentos necessários para o levantar, determina que essa ação sofra maior influência de alterações no peso, principalmente, se forem provenientes de incrementos na gordura corporal. Outro aspecto a considerar é que, sendo o TSL um procedimento simples de rastreamento, pode ter um efeito teto, não ocorrendo discriminação nos valores mais altos. Dessa forma, pode ser que o sobrepeso prejudique o desempenho, mas como os indivíduos já realizavam o teste com facilidade, não chegou a haver redução do escore.

Parece que indivíduos fisicamente ativos apresentam o desempenho comprometido, na ação de levantar do solo, somente quando o sobrepeso é caracterizado, embora isso não consista em uma condição obrigatória. Os soldados no estudo 1 experimentaram dificuldade significativa com $20 \%$ de incremento no peso (A0 x A20), situação em que tanto o IMC quanto o \%g caracterizavam a condição de sobrepeso.

Merecendo atenção, os soldados usualmente realizam treinamentos e manobras carregando equipamentos que, somados, tendem a exceder a sobrecarga de $10 \%$ do peso corporal. Porém, como os mesmos são treinados para tal, e nessas circunstâncias o peso extra não se encontra exclusivamente no tronco, diferentemente do simulado em nosso estudo, não é possível fazer inferências envolvendo reduções no desempenho durante as manobras. Contudo, parece que os soldados que se encontrarem com peso corporal elevado, mesmo ainda não caracterizando o sobrepeso, tendem a enfrentar maiores dificuldades.

No estudo 2, entretanto, foram observados resultados ligeiramente distintos. Diferenças significativas foram evidenciadas com $30 \%$ de aumento no peso corporal (A30) ( $\mathrm{p}<0,05)$, embora incrementos de $10 \%$ e $20 \%$ já resultassem em sobrepeso, considerando o IMC, isoladamente. Vale ressaltar que, apesar de o nível de atividade física semanal ser superior nos jogadores, é improvável que isso justifique as diferenças observadas, uma vez que os exercícios semanais realizados eram predominantemente aeróbios e, portanto, pouco específicos para o desempenho no teste.

Os resultados dos estudos conjuntamente demonstram que parece haver um limite de incremento no peso corporal, por volta de 20 a $30 \%$, a partir do qual o desempenho nas ações é comprometido. Em outras palavras, a queda do desempenho na ação de levantar parece ocorrer próxima à condição de sobrepeso, podendo ainda piorar caso haja aumentos subseqüentes na massa corporal.

Verificou-se que para a ação de sentar, no primeiro estudo, dois indivíduos foram capazes de manter o desempenho máximo, enquanto seis apresentaram o mesmo resultado em relação ao levantar. Já no estudo 2, observou-se o mesmo em seis indivíduos, tanto para o sentar, quanto para 
do e artificial no peso corporal. Nesse sentido, não se pode garantir que as pessoas com sobrepeso experimentariam limitações no desempenho em sentar e levantar do solo equivalentes às evidenciadas em nossos estudos. Contudo, considerando que as amostras incluíam atletas jovens e homens fisicamente ativos, é possível que os mesmos incrementos no peso corporal resultem em maiores limitações na população em geral. Dados epidemiológicos sugerem que cerca de $2 / 3$ das populações norte-americana, australiana, canadense, britânica e do Estado de São Paulo, no Brasil, são sedentárias ${ }^{33,34}$.

Os achados aqui descritos compartilham da mesma lógica que permeia a perda de força com o envelhecimento. Há um consenso de que o processo de sarcopenia, ou seja, a redução progressiva de massa muscular com a elevação da idade, independentemente do peso corporal, acarreta maior esforço para a realização das atividades do cotidiano ${ }^{35,36}$. O aumento no percentual de gordura em jovens acaba por antecipar tal situação, uma vez que a proporção de massa corporal magra no peso total diminui, conforme a adiposidade aumenta. Esta talvez seja uma das razões para o baixo perfil de atividade física nesta população, já que as mesmas atividades acabam por demandar maior esforço físico para serem conduzidas.

\section{REFERÊNCIAS}

1. National Institute of Diabetes and Digestive and Kidney Diseases. Understanding Adult Obesity. NIH Publ. n 94-3680. Rockville: National Institutes of Health, 1993.

2. Kukszmarski RJ, Flegal MK, Campbell SM, Johnson CL. Increasing prevalence of overweight among U.S. adults: The National Health and Nutrition Examination Surveys. JAMA 1994;272:205-11.

3. Kurscheid T, Lauterbach K. The cost implications of obesity for health care and society. Int J Obes Relat Metab Disord 1998;22(Suppl 1):S3S6.

4. Flegal KM. Trends in body weight and overweight in the U.S. population. Nutr Rev 1996;54:S97-S100.

5. Birmingham CL, Muller JL, Palepu A, Spinelli JJ, Anis AH. The cost of obesity in Canada. CMAJ 1999;160:483-8.

6. Zanella MT. Obesidade. In: Mion Jr. D, Nobre F, editors. Risco cardiovascular global. São Paulo: Lemos Editorial 1999;103-14.

7. Stern M. Epidemiology of obesity and its link to heart disease. Metab Clin Exp 1995;44:S1-S3.

8. Hardman AE. Physical activity, obesity and blood lipids. Int J Obes Relat Metab Disord 1999;23(Suppl 3):S64-S71.

9. Matsushima M, Kriska A, Tajima N, La-Porte R. The epidemiology of physical activity and childhood obesity. Diabetes Res Clin Pract 1990; 10:S95-S102.

10. Maffeis C, Zaffanello M, Schutz Y. Relationship between physical inactivity and adiposity in prepubertal boys. J Pediatr 1997;131:288-92.

11. Fleming BE, Wilson DR, Pendergast DR. A portable, easily performed muscle power test and its association with falls by elderly persons. Arch Phys Med Rehabil 1991;72:886-9.

\section{CONCLUSÃO}

Aumentos no peso corporal, simulados artificialmente pelo uso de coletes de areia no tronco, tendem a comprometer o desempenho nas ações de sentar e levantar do solo, em adolescentes e adultos jovens fisicamente ativos. Os soldados enfrentam limitações no levantar assim que a condição mínima para sobrepeso é evidenciada. Por outro lado, os jogadores de futebol adolescentes somente as experimentam em condição de elevado sobrepeso. Paralelamente, comprometimentos na ação de sentar talvez só ocorram em IMC em níveis de obesidade.

As dificuldades encontradas por indivíduos fisicamente ativos em nosso estudo apontam que os indivíduos com sobrepeso real possivelmente apresentam desempenhos relativamente limitados em ambas as ações, já que baixos níveis de atividade física são comuns nessa população. Estudos ainda são necessários para confirmar esses aspectos. Esses mesmos ensaios devem procurar delimitar melhor a influência da flexibilidade específica, nos movimentos de flexão e adução do quadril, sobre o desempenho no TSL em indivíduos com sobrepeso corporal e obesidade.

\section{AGRADECIMENTOS}

Este estudo foi parcialmente fomentado pela Capes.

12. Linden DWV, Brunt D, McCulloch MU. Variant and invariant characteristics of the sit-to-stand task in healthy elderly adults. Arch Phys Med Rehabil 1994;75:653-60.

13. Schenkman M, Hughes MA, Samsa G, Studenski S. The relative importance of strength and balance in chair rise by functionally impaired older individuals. J Am Geriatr Soc 1996;44:1441-6.

14. Araújo CGS. Teste de Sentar-Levantar - Apresentação preliminar de um procedimento para avaliação em Medicina do Exercício e do Esporte. Rev Bras Med Esporte 1999;5:179-82.

15. Araújo CGS. Teste de Sentar-Levantar - Um instrumento para rastreamento em Medicina do Exercício e do Esporte. Âmbito Medicina Desportiva $1999 ; 59: 18-20$.

16. Lira VA, Araújo CGS. Teste de Sentar-Levantar: estudos de fidedignidade. Revista Brasileira de Ciências do Movimento 2000 (no prelo).

17. Tanner JM. Growth of adolescents. $2^{\text {nd }}$ ed. Oxford, UK: Blackwell Scientific, 1962.

18. Jackson AS, Pollock ML. Generalysed equations for predicting body density in men. Br J Nutr 1978;40:497-504.

19. Siri WE. Body composition from fluid spaces and density. In: Brozek J, Henzel A, editors. Techniques for measuring body composition. Washington DC: National Academy of Science, 1969;223-44.

20. Heath BH, Carter JEL. A modified somatotype method. Am J Phys Anthrop 1967;27:57-74.

21. Araújo CGS. Dez anos do somatótipo Heath-Carter no Brasil - Um posicionamento crítico. In: Araújo CGS, editor. Fundamentos biológicos: medicina desportiva. Rio de Janeiro: Ao Livro Técnico, 1985;65-88.

22. Pollock ML, Wilmore JH. Exercise in health and disease: evaluation and prescription for prevention and rehabilitation. $2^{\text {nd }}$ ed. Philadelphia: WB Saunders, 1990. 
23. Araújo CGS. Avaliação e treinamento da flexibilidade. In: Ghorayeb N, Barros Neto TL, editors. O exercício. São Paulo: Atheneu, 1999;25-34

24. Araújo CGS. Flexiteste - Uma nova versão dos mapas de avaliação. Kinesis 1986;2:251-67.

25. Lira VA, Khouri RA, Farinatti PTV, Araújo, CGS. Influence of some morphological and functional variables on the performance of sitting and rising from the floor. Journal of Athletic Training (submetido para publicação).

26. Havlicek I, Cechvala J. The study of adequate running distance for the different age groups. Prague: Charles University Press, 1969.

27. Sayers PS, Harackiewicz DV, Harman EA, Frykman PN, Rosenstein MT Cross-validation of three jump power equations. Med Sci Sports Exerc 1999;31:572-7.

28. Campbell TD, Stanley CJ. Delineamentos experimentais e quase experimentais de pesquisa. São Paulo: Editora da Universidade de São Paulo, 1979.

29. Hill JO, Drougas HJ, Peters JC. Physical activity, fitness, and moderate obesity. In: Bouchard C, Shephard RJ, Stephens T, editors. Physical activity, fitness, and health: international proceedings and consensus statement. Champaign: Human Kinetics, 1994;684-95.
30. Sichieri R, Allam VLC. Avaliação do estado nutricional de adolescentes brasileiros através do índice de massa corporal. Jornal de Pediatria 1996; 72:80-4.

31. Guo SS, Roche AF, Cameron Chumlea W, Gardner JD, Siervolgel RM. The predictive value of childhood body mass index values for overweight at age 35 yr. Am J Clin Nutr 1994;59:810-9.

32. Bar-Or O, Foreyt J, Bouchard C, Brownell KD, Dietz WH, Ravussin E, et al. Physical activity, genetic, and nutritional considerations in childhood weight management. Med Sci Sports Exerc 1998;30:2-10.

33. Stephens T, Capersen CJ. The demography of physical activity. In: Bouchard C, Shephard RJ, Stephens T, editors. Physical activity, fitness, and health: international proceedings and consensus statement. Champaign: Human Kinetics, 1994;204-13.

34. Forjaz CLM, Negrão CE. Sedentarismo. In: Mion Jr. D, Nobre F, editors. Risco cardiovascular global. São Paulo: Lemos Editorial, 1999;13962 .

35. Larsson I. Histochemical characteristics of human skeletal muscle during aging. Acta Physiol Scand 1983;117:469-71.

36. Mazzeo RS, Cavanagh P, Evans W J, Fiatarone M, Hagberg J, McAuley E, et al. Exercise and physical activity for older adults. Med Sci Sports Exerc 1998;30:992-1008. 